Artículo Original. Mayo-Agosto 2017; 7(2):60-67. Recibido: 16/01/2017 Aceptado: 09/04/2017.

http://dx.doi.org/10.21929/abavet2017.72.5

\title{
Utilización de aceites ozonizados para el tratamiento de heridas en bovinos de engorda
}

\author{
Use of ozonized oils for the treatment of wounds in fattening bovine
}

\author{
Peña-Jiménez Francisco ${ }^{1 *}$ fjpenj@hotmail.com, Zamora-Rodríguez Zullyt ${ }^{2}$ \\ efleitas@infomed.sld.cu, Hernández-Aco Rodrigo' salomon.kaleb@hotmail.com, Fleitas- \\ González Eduardo³ efleitas@infomed.sld.cu, Hernández-González Adrían ${ }^{1}$ \\ adrianhy10@hotmail.com, Hernández-Rosado Abigail4 abidu71@hotmail.com
}

1Universidad Autónoma del Estado de Hidalgo. Instituto de Ciencias Agropecuarias. Tulancingo de Bravo, Hidalgo. México. 2Departamento de Farmacología, Unidad de Sustancias Ozonizadas y Productos Naturales, Centro Nacional de Investigaciones Científicas de Cuba. 3Clínico Privado. Director de la Clínica Veterinaria "Almiqui", La Habana, Cuba. 4Clínico Privado. *Autor responsable y de correspondencia: PeñaJiménez Francisco. Universidad Autónoma del Estado de Hidalgo. Instituto de Ciencias Agropecuarias. Rancho Universitario. Av. Universidad Km. 1. Ex Hacienda Aquetzalpa, Apartado Postal No. 32, Tulancingo de Bravo, Hidalgo.

\section{RESUMEN}

El cierre de heridas cutáneas está compuesto por una compleja organización de los tejidos, que incluyen hemorragia, inflamación, re-epiteliziación, formación de tejido de granulación, fases finales de la reparación y remodelación. Hoy en día, es práctico utilizar el $\mathrm{O}_{3}$ en combinación con aceites vegetales como aceite de oliva, lo que da como resultado aceite de oliva ozonizado. El aceite ozonizado se utiliza para el tratamiento de heridas crónicas, pero ha habido pocos estudios relacionados con los efectos terapéuticos del aceite de oliva ozonizado en cicatrización aguda. La finalidad de este trabajo fue probar la eficacia de aceites ozonizados en la cicatrización de heridas cutáneas en bovinos de engorda. A 10 bovinos de engorda se les realizaron tres incisiones quirúrgicas circulares en la tabla del cuello, que fueron tratadas diariamente con aceites ozonizados, aceite ozonizado de oliva (T1), aceite ozonizado comercial (Oleozon®) (T2) y un control negativo. La cicatrización se evaluó diariamente. Los bovinos tratados con aceite de oliva ozonizado tuvieron una menor producción de costra, las heridas estaban humectas y con escasa retracción de la epidermis, respecto a los controles negativos. Concluyendo que el aceite de oliva ozonizado aceleran el proceso de cicatrización en los bovinos de engorda.

Palabras clave: Ozono, aceites ozonizados, heridas, bovinos.

\begin{abstract}
The closure of cutaneous wounds is a complex system of tissues organization that includes hemorrhage, inflammation, re-epithelialization, the formation of granulation tissue and the final stages of repair and remodeling. Today, it is practical and cost-effective to use $\mathrm{O} 3$ in combination with vegetable oil like olive oil to obtain ozonized oils. Ozonized oils have been used topically for the treatment of chronic wounds. However, exist few investigations related to the therapeutic effects on acute healing. The purpose of this study was to evaluate the efficacy of ozonated oils in the healing of skin wounds in fattening bovines. Ten bovines were submitted to three circular surgical incisions in the neck table, which were treated daily with ozonated olive oil (T1) and commercial ozonized oil (Oleozon®) (T2). One surgical incision was used as negative control. The effect of daily cicatrization was evaluated, finding that the bovine treated with ozonated olive oils had a lower production of scab. Also, the wounds were humid and with a little retraction of the
\end{abstract}


epidermis in comparison with the negative controls. Concluding that ozonized olive oil accelerates the healing process in fattening bovines.

Keywords: Ozone, ozonized oils, wounds, bovine

\section{INTRODUCCIÓN}

El cierre de heridas cutáneas está compuesto por una compleja organización de los tejidos, que incluyen la hemorragia, inflamación, re-epiteliziación, formación de tejido de granulación y las fases finales de la reparación y la remodelación (Kimet al., 2009). Esta compleja organización incluye la coordinación de docenas de tipos de células y proteínas de la matriz, que son importantes para controlar las etapas del proceso de la reparación. Estudios previos han demostrado que los factores de crecimiento endógenos, tales como factores de crecimiento de los fibroblastos (FGF) (Ornitz y Itoh, 2001), factores de crecimiento derivados de las plaquetas (PDGF) (Heldin y Westermark, 1999), factores de crecimiento $\beta$ (TGF- $\beta$ )(Werner y Grose, 2003) y factores de crecimiento del endotelio vascular (VEGF) (Lauer et al., 2000), son polipéptidos reguladores del proceso de curación, que están relacionados con la liberación de macrófagos, fibroblastos y queratinocitos en el sitio de la lesión que participan en la regulación de la reepitelización, formación de tejido de granulación, síntesis de colágeno y neovascularización (Kim et al., 2009).

El ozono $\left(\mathrm{O}_{3}\right)$ ha sido ampliamente reconocido como uno de los mejores bactericidas y antifúngicos (Al-Dalain et al., 2001; Valacchi et al., 2005; Elvis y Ekta, 2011) y se ha utilizado empíricamente como un agente terapéutico en el tratamiento de heridas, tales como úlceras tróficas, úlceras isquémicas y en heridas de diabéticos (Martínez-Sánchez et al., 2005; De Monte et al., 2005; Elvis y Ekta, 2011). Los efectos beneficiosos del $\mathrm{O}_{3}$ en la cicatrización de heridas se ha atribuido a la disminución de la carga bacteriana en la herida infectada y al aumento de la tensión de oxígeno por la exposición $\mathrm{O}_{3}$ en el área de la herida (Gajendrareddy et al., 2005; Lim et al., 2006).

El ozono es un fuerte agente oxidante y posee propiedades bactericidas; éste puede actuar como un inductor de citosinas (Bocci et al., 1993), tales como interferón (IFN- $\gamma$ y $\beta$ ), factor de necrosis tumoral (FNT- $\alpha$ ) interleucinas (IL) 1 $\beta, 2,4,6,8$ y 10, factor estimulador de colonia granulocito-macrófago (GM-CSF) y el factor transformador del crecimiento (TGF- $\beta 1$ ) (Bocci et al., 1994).

Hoy en día, es práctico y rentable utilizar el $\mathrm{O}_{3}$ en combinación con aceites vegetales, como es el caso del aceite de oliva, obteniendo aceite de oliva ozonizado. Los aceites ozonizados contienen la molécula de $\mathrm{O}_{3}$ estabilizada, como un ozónido entre los enlaces dobles de un ácido graso monoinsaturado como el ácido oleico, que es ideal para el uso tópico del $\mathrm{O}_{3}$ en heridas cutáneas crónicas e infectadas (Valacchi et al., 2005). 
El aceite ozonizado se ha utilizado tópicamente para el tratamiento de heridas crónicas, pero ha habido pocos estudios relacionados con los efectos terapéuticos del aceite de oliva ozonizado en la cicatrización aguda (Kimet al., 2009).

Durante la última década, han sido objeto de publicación diversos efectos beneficiosos del pre-condicionamiento oxidativo con Ozono (POO) (Ajamieh et al., 2004; Borrego et al., 2004), el cual, aplicado con un criterio adecuado, se define como una adaptación ante un estrés oxidativo agudo o pre-condicionamiento oxidativo (Bocci, 2006; Zamora et al., 2007; Zamora et al., 2008). También se ha demostrado que la ozonoterapia restablece el balance redox intracelular; incrementando la actividad de las enzimas antioxidantes endógenas, y de esta forma disminuye el estrés oxidativo generado en cualquier proceso patológico (Borrego et al., 2004; Zamora et al., 2005).

Por lo cual, el objetivo de este estudio fue evaluar el efecto de los aceites vegetales ozonizados sobre la cicatrización en heridas cutáneas agudas en bovinos de engorda.

\section{Bovinos de engorda}

\section{MATERIAL Y MÉTODOS}

El presente trabajo se realizó con diez bovinos machos enteros, mestizos, con un peso promedio de 405.85 kilogramos y con una edad promedio de 15 meses.

\section{Ozonización del aceite de oliva}

Se empleó aceite de oliva extra virgen comercial, el cual contiene $78.5 \%$ de grasas monoinsaturados (omega 9), $7.1 \%$ de grasas poli instaurados (Omega 3 y 6 ) y $14 \%$ de grasas saturadas. Se ozonizaron $100 \mathrm{ml}$ del aceite de oliva, con un ozonizador de aceite de dos potencias (OzonoBio3, Modelo AO-5); se burbujeó durante 1 h una concentración de ozono de $50 \mathrm{mg} / \mathrm{ml}$, el cual estaba conectado a un generador de oxígeno (Respironics Everflo, Philips) generando un flujo de oxígeno de $2 \mathrm{~L} / \mathrm{min}$.

Para dicho estudio se empleó el aceite de oliva ozonizado antes mencionado, además de un aceite de girasol ozonizado comercial OLEOZON®.

\section{Determinación del índice de peróxidos}

El índice de peróxido se determinó empleado la tecina reportada por Farmacopedia Británica (British Parmacopeia, 2000). Se evaluó el índice de peróxido para el aceite de oliva ozonizado y OLEOZON®. Para su realización se colocaron $5 \mathrm{~g}$ de la muestra en un matraz con $30 \mathrm{ml}$ de una solución ácido acético-cloroformo 2:3. La muestra se agitó hasta disolverse totalmente y se le adicionó $0.5 \mathrm{ml}$ de una solución saturada de yoduro de potasio y se dejó reposar por un minuto. Al finalizar el tiempo se le adicionaron $30 \mathrm{ml}$ de agua destilada y procedió a titularse con tiosulfato de sodio $0.01 \mathrm{~N}$ hasta obtener una coloración ligeramente amarilla. Después de obtener dicha coloración, se le adicionó 0.5 $\mathrm{ml}$ de una solución indicadora de almidón y se continuó titulando hasta la aparición de un color azul. Bajo las mismas condiciones se realizó la titulación de un blanco. 
El índice de peróxido se expresó en mili equivalentes de peróxido en un kg de aceite y se calculó empleando la siguiente formula.

$$
\text { I. } P=\frac{\left(A-A_{1}\right) * N * 1000}{M}
$$

I.P = Índice de Peróxido

$A=$ Mililitros de solución de tiosulfato de sodio gastados en la titulación de la muestra.

$\mathrm{A} 1=\mathrm{ml}$ de solución de tiosulfato de sodio gastados en la titulación del blanco.

$\mathrm{N}=$ Normalidad de la solución de tiosulfato de sodio.

$\mathrm{M}=$ Masa de la muestra en gramos.

El índice de peróxido encontrando para el aceite ozonizado de oliva, estuvo en un rango de 400 to $490 \mathrm{mmol} / \mathrm{kg}$ y el aceite ozonizado comercial, OLEOZON® en un rango de 500 to $800 \mathrm{mmol} / \mathrm{kg}$.

\section{Preparación de los animales experimentales}

Se realizó un examen físico general a cada uno de los animales experimentales, en el cual no se encontró ninguna anormalidad; enseguida se preparó la tabla del cuello derecha para la intervención quirúrgica. Los animales fueron anestesiados localmente con lidocaína al $2 \%$. A cada paciente se le hicieron tres incisiones quirúrgicas circulares en la tabla del cuello, con un diámetro de $2 \mathrm{~cm}$ y una profundidad de $3 \mathrm{~mm}$.

La intervención se realizó con los animales en pie, razón por la que no se utilizaron tranquilizantes para evitar decúbitos accidentales durante la manipulación de los mismos.

\section{Tratamiento experimentales}

Las heridas de los bovinos en engorda fueron monitoreadas cada $24 \mathrm{~h}$. Cada una de las heridas realizadas recibió un tratamiento diferente, dos de ellas se trataron de manera tópica con los aceites ozonizados; para el tratamiento 1 (T1) se usó el aceite de oliva ozonizado y para el tratamiento 2 (T2) se usó el aceite ozonizado comercial OLEOZON®. Una de las heridas (control negativo) no recibió ningún tratamiento.

La duración del tratamiento fue de quince días, el cual consistió en la limpieza diaria de cada una de las heridas y la aplicación tópica de estos aceites cada veinticuatro horas.

\section{RESULTADOS Y DISCUSIÓN}

En la Tabla 1 se observan lo signos más relevantes de la cicatrización respecto al tiempo y tratamiento. 


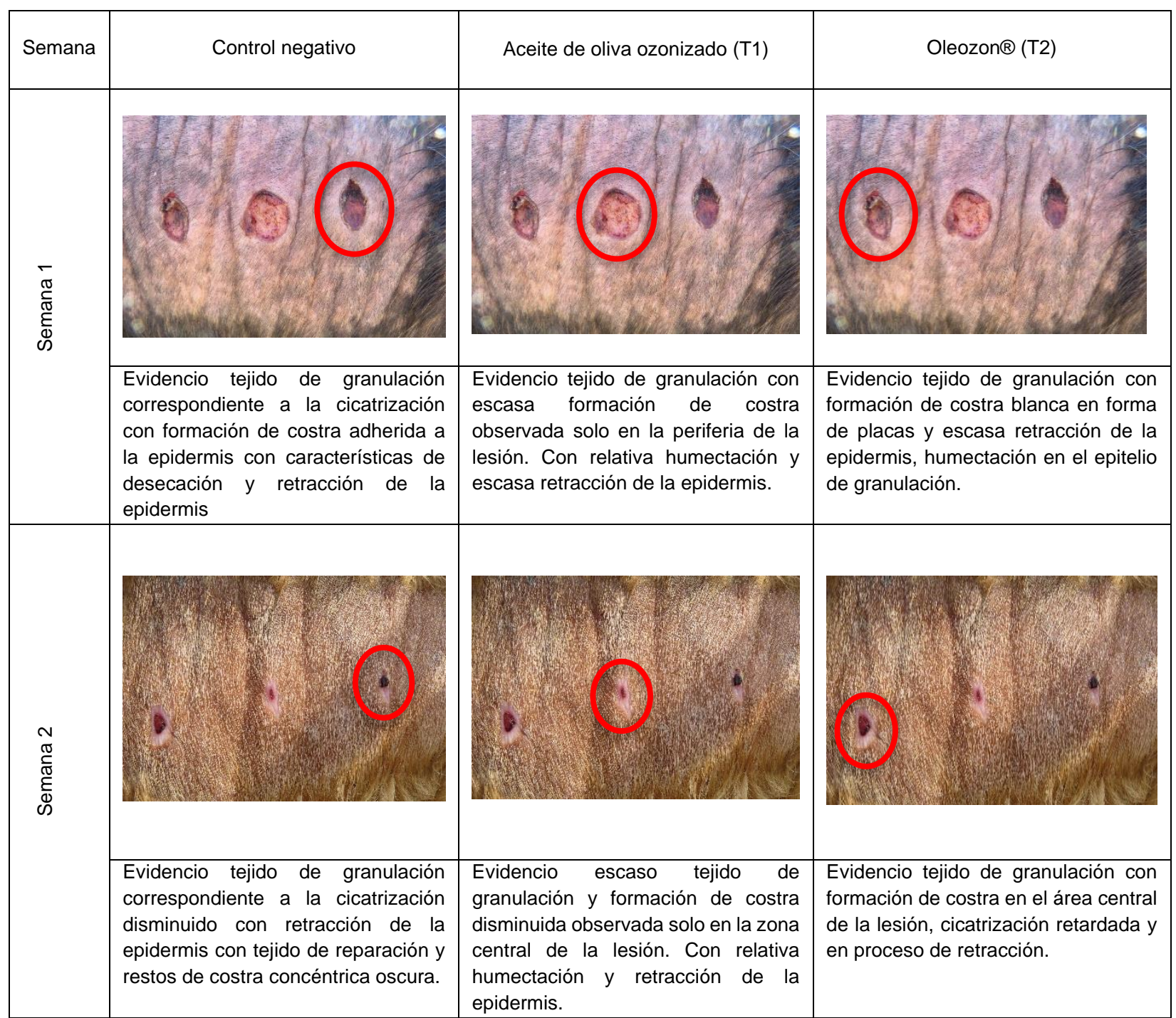

Tabla 1. Evaluación macroscópica de la cicatrización de las heridas cutáneas en bovinos de engorda.

Se observó de manera general que la cicatrización de heridas fue más rápida cuando se empleó el aceite de oliva ozonizado, esto puede atribuirse a las propiedades germicidas, de restauración de tejido e inmune-estimulantes de los aceites ozonizados (MartínezSánchez et al., 2012). Además, cuando se emplearon los aceites ozonizados se observaron que las heridas se encontraban más humectadas respecto al control y se sabe que las heridas en un entorno húmedo, tiene una mayor epitelización, lo cual influye en el tiempo de recuperación (Bigbie et al., 1989). El mecanismo de acción exacto sobre 
la actividad biológica de los aceites ozonizados es aún desconocido; sin embargo, existen varias hipótesis que intentan explicarlo. Una de ellas plantea que es probable que los triozonidos estables al entrar en contacto con los exudados de las heridas, que se encuentran a una temperatura de aproximada de $37^{\circ} \mathrm{C}$, provoca que se descompongan y generen ozono, el cual forma peróxido de hidrógeno y lipoperóxidos, que son los responsables de los efectos regenerativos y desinfectantes (Bocci, 2005; MartínezSánchez, et al., 2012).

También se plantea que la liberación lenta de ozono en las heridas favorece el proceso de cicatrización, no sólo por la desinfección local, sino también por favorecer a nivel local la liberación de citosinas con efectos reparadores (Valacchi et al., 2005; Valacchi et al., 2011).

\section{CONCLUSIÓN}

Los aceites vegetales ozonizados aceleraron el proceso de cicatrización de las heridas cutáneas de los bovinos en engorda. Las heridas tratadas con aceite de oliva ozonizado, mostraron mayor retracción de la epidermis, con respecto a los controles negativos y a los tratados con el aceite comercial. Las heridas tratadas con el aceite ozonizado comercial (OLEOZON®), tuvieron una cicatrización más tardada, con respecto a las tratadas con aceite de oliva ozonizado.

\section{LITERATURA CITADA}

AJAMIEH HH, Menéndez S, Martinez-Sanchez G, Candelario-Jalil E, Re L, Giuliani A and Fernández OS. 2004. Effects of ozone oxidative preconditioning on nitric oxide generation and cellular redox balance in a rat model of hepatic ischemia-reperfusion. Liver Int. 24(1):55- 62. ISSN: 1478-3231. DOI: 10.1111/j.1478-3231.2004.00885.x

AL-DALAIN SM, Martinez G, Candelario-Jalil E, Menendez S, Re L, Giuliani A, Leon OS. 2001. Ozone treatment reduces markers of oxidative and endothelial damage in an experimental diabetes model in rats. Pharmacol Res. 44(5):391-6. ISSN: 1043-6618.

DOI: $10.1006 /$ phrs.2001.0867

BIGBIE RB, Schumacher J, Moll D, Engelbert T. 1989. Equine amnion as a biological dressing in the treatment of open wounds in horses. Proceedings. Am. Associ. Equine pract. 117-125. ISSN: 17498848

BOCCI V, Luzzi E, Corradeschi F, Paulesu L, Rossi R, Cardaioli E, Di Simplicio P. 1993. Studies on the biological effects of ozone: 4 Cytokine production and glutathione levels in human erytrocytes. J Biol Regul Homeost Agents. 7:133-138. ISSN: 0393-974X/2017

BOCCI V, Luzzi E, Corradeschi F, Silvestri S. 1994. Studies on the biologycal effects of ozone: Producction of transformaning growth factor $\beta$ by human blood after ozone treatment. J Biol Regul Homeost Agents. 8:108- 111. ISSN: 0393-974X/2017 
BOCCI V. 2006. Is true that ozone is always toxic? The end of a dogma. Toxicology and Applied Pharmacology. 1; 216(3):493-504. ISSN: 0041-008X. DOI: 10.1016/j.taap.2006.06.009

BOCCI V. 2005. Ozone A New Medical Drug. Dordrecht, The Nederlands: Springer. 295 p. ISBN-13: 978-1402031397

BORREGO A, Zamora ZB, González R, Romay Ch, Menéndez S, Hernández F, Montero T, Rojas E. 2004. Protection by ozone preconditioning is mediated by the antioxidant system in cisplatin-induced nephrotoxicity in rats. Mediat Inflamm. 13(1):13-19. ISSN: 1466-1861. DOI: 10.1080/09629350410001664806

BRITISH Pharmacopoeia. 2000. Appendix XF, IA, IB. Peroxide value. http://www.uspbpep.com/bp2008/data/899.asp

DE MONTE A, van der Zee H, Bocci V. 2005. Major ozonated autohemotherapy in chronic limb ischemia with ulcerations. J Altern Complement Med. 11(2):363-7. ISSN: 1075-5535. DOI: $10.1089 / \mathrm{acm} .2005 .11 .363$

ELVIS AM, Ekta JS. 2011. Ozone therapy: A clinical review. Journal of Natural Science, Biology and Medicine. 2(1):66-70. ISSN: 0976-9668. DOI: 10.4103/0976-9668.82319

GAJENDRAREDDY PK, Sen CK, Horan MP, Marucha PT. 2005. Hyperbaric oxygen therapy ameliorates stress-impaired dermal wound healing. Brain Behav Immun. 19(3): 217-22. ISSN: 0889-1591. DOI: 10.1016/j.bbi.2004.09.003

HELDIN CH, Westermark B. 1999. Mechanism of action and in vivo role of platelet-derived growth factor. Physiol Rev. 79(4):1283-316. ISSN: 0031-9333.

KIM HS, Noh SU, Han YW, Kim KM, Kang H, Kim HO, Park YM. 2009. Therapeutic Effects of Topical Application of Ozone on Acute Cutaneous Wound Healing.J Korean Med Sci. 24 (3): 368-374. ISSN: 1011-8934. DOI: 10.3346/jkms.2009.24.3.368

LAUER G, Sollberg S, Cole M, Flamme I, Sturzebecher J, Mann K, Krieg T, Eming SA. 2000. Expression and proteolysis of vascular endothe- lial growth factor is increased in chronic wounds. J Invest Dermatol. 115(1):12-8. ISSN: 0022-202X. DOI: 10.1046/j.15231747.2000.00036.x

LIM Y, Phung AD, Corbacho AM, Aung HH, Maioli E, Reznick AZ, Cross CE, Davis PA, Valacchi G. 2006. Modulation of cutaneous wound healing by ozone:differences between young and aged mice. Toxicol Lett. 160(2):127-34. ISSN: 0378-4274. DOI: 10.1016/j.toxlet.2005.06.013

MARTÍNEZ-SÁNCHEZ G, Al-Dalain SM, Menéndez S, Re L, Giuliani A, Candelario-Jalil E, Álvarez H, Fernández-Montequin JI, León OS. 2005. Therapeutic efficacy of ozone in 
patients with diabetic foot. Eur J Pharmacol. 523(1-3):151-61. ISSN: 0014-2999. DOI: 10.1016/j.ejphar.2005.08.020.

MARTíNEZ-SÁNCHEZ G, Re L, Pérez-Davison G, Horwat Delaporte R. 2012. Las aplicaciones médicas de los aceites ozonizados, actualización. Revista Española de Ozonoterapia. 2(1):121-139. ISSN: 2174-3215

ORNITZ DM, Itoh N. 2001. Fibroblast growth factors. Genome Biol. 2(3): 3005. ISSN: 1474-760X. DOI: 10.1186/gb-2001-2-3

VALACCHI G, Fortino V, Bocci V. 2005. The dual action of ozone on the skin. Br J Dermatol. 153(6):1096-100. ISSN: 1365-2133. DOI: 10.1111/j.1365-2133.2005.06939.x

VALACCHI G, Lim Y, Belmonte G, et al. 2011. Ozonated sesame oil enhances cutaneous wound healing in SKH1 mice. Wound Repair Regen. 19(1):107-15. ISSN: 1524-475X. DOI: $10.1111 / \mathrm{j} .1524-475 X .2010 .00649 . x$

WERNER S, Grose R. 2003. Regulation of wound healing by growth factors and cytokines. Physiol Rev. 83:835-70. ISSN: 0031-9333. DOI: 10.1152/physrev.00031.2002

ZAMORA-RODRíGUEZ ZB, González Álvarez R, Guanche D, Merino N, Hernández RF, Menéndez CS, Alonso GY, Schulz S. 2007. Antioxidant mechanism is involved in the gastroprotective effects of ozonized sunflower oil in ethanol-induced ulcers in rats. Mediators Inflamm. 2007(65873). ISSN: 1466-1861. DOI: 10.1155/2007/65873

ZAMORA Z, González R, Guanche D, et al. 2008. Ozonized sunflower oil reduces oxidative damage induced by indomethacin in rat gastric mucosa. Inflamm Res. 57(1): 3943. ISSN: 1023-3830. DOI: 10.1007/s00011-007-7034-1

ZAMORA ZB, Borrego A, López OY, Delgado R, González R, Menéndez S, Hernández F, Schulz S. 2005. Effects of ozone oxidative Preconditioning on TNF- alpha release and antioxidant-prooxidant intracellular balance in mice during endotoxic shock. mediators inflamm. (1):16-22. ISSN: 1466-1861. DOI: 10.1155/MI.2005.16

\section{AGRADECIMIENTOS}

Los autores de este artículo agradecen a la Universidad Autónoma del Estado de Hidalgo y al Programa para el Desarrollo Profesional Docente, para el Tipo Superior (PRODEP) por el apoyo facilitado para la realización de este trabajo. 